\title{
Oral Bioavailability and Neuro-Availability vs. "Neuro-Transmittability": A Figment of Imagination in the Conundrum of Neuronal Intervention
}

\section{Viness Pillay ${ }^{1 *}$, Pradeep Kumar ${ }^{1}$, Yahya E Choonara ${ }^{1}$ and Girish Modi ${ }^{2}$}

${ }^{1}$ University of the Witwatersrand, Faculty of Health Sciences, Department of Pharmacy and Pharmacology, 7 York Road, Parktown, 2193 , Johannesburg, South Africa ${ }^{2}$ University of the Witwatersrand, Faculty of Health Sciences, Division of Neurosciences, Department of Neurology, 7 York Road, Parktown 2193, Johannesburg, South Africa

Quantification of the neuro-availability of drugs that are administered directly to the central nervous system (CNS) or via the oral or parenteral routes is important for determining the effect of these drugs in treating several neurodegenerative disorders such as Alzheimer's disease, Parkinson's disease or traumatic and nontraumatic cerebral injuries. The neuro-availability of drug after oral administration is dependent on two crucial factors such as 1) the absorption of the drug from the gastrointestinal tract, and 2) the hydrophilic-lipophilic balance of the drug molecule to penetrate the restrictive barriers of the CNS. The latter factor is, in addition, very significant when the drug is administered via the parenteral route [1].

Combining these established proclamations, it is typically proposed that in order to achieve enhanced neuro-availability, the conventional oral bioavailability of the drug should be enhanced. Quantification of the oral bioavailability and subsequent neuroavailability of the administered drug may mislead the neurotherapeutic efficacy as a prediction. The activity of peripherally-acting neuroactive drugs is higher with an increase in oral bioavailability since they produce their biological effect via direct interaction with the target receptors. However, in the case of neuro-interventional paradigms involving centrally-acting neuroactive drugs, the conundrum after oral drug administration to neuro-biological effect follows a much more complex biochemical pathway. The variation of the solubilitypermeability aspect affects the absorption of the drug molecules via the gastrointestinal tract and this forms the basis of the oral bioavailability profile of the drug. The oral bioavailability further influences the neuro-availability of the drug as an increase in neuroavailability is directly proportional to an increase in oral bioavailability given that the lipophilic-hydrophilic conditions are well addressed with respect to the passage through the Blood Brain Barrier (BBB). The entry of a drug into the brain extracellular fluid leads to a series of events involving the drug specific and site specific target receptor interactions followed by the release or reuptake inhibition of neuromediators and neurotransmitters resulting in the neurological action important to the homeostasis and the underlying pathological conditions [2]. It is evident from the above discussion that an increase in oral bioavailability may not guarantee an increase in neuro-availability as this is controlled by the BBB which further limits the neuro-availability of the drug based on various physicochemical properties.

Therefore determining the oral bioavailability of a drug may not necessarily correlate well with the neurological response, leading to the need for quantifying the "exact" neuro-availability. Furthermore, a highly neuro-available drug may also not pass through all the restrictive barriers of the CNS such as Brain Capillary Endothelial Cells, the Choroid Plexus, Cuboidal Epithelium, and the Endothelial Efflux Transporters prevalent throughout the cerebral epithelium. In the best case scenario, even if the drug succeeds to bypass the underlying cerebral barriers, the effect of influx or efflux transport mechanisms bordering the $\mathrm{BBB}$ and the far reaching blood-CSF and conversely CSF-brain parenchyma interfaces will result in the establishment of the "drug-dependent heterogeneity of concentration ratios among cerebral extracellular space compartments" [3]. Hence, these drugs may not be able to excite or inhibit target neuroreceptors responsible for the release or non-reuptake of neurotransmitters and neuromediators.

We propose that instead of measuring the oral bioavailability or neuro-availability of the administered drugs, pharmaceutical and neuroscience researchers should focus on quantifying the "NEUROTRANSMITTABILITY" of the administered neuroactive drugs. The neurotherapeutic effect of various drugs leads to the release of neurotransmitters or neuromodulators such as serotonin. This may further define and influence the underlying neurological mechanisms. Considering the serotonin-based biochemical pathways, neurotransmission via serotonin is highly complex given that there are 15 different sub-types of serotonin receptors isolated in different regions of the brain and are protected by their own efflux transporters [4]. For example, with regard to the median raphe nucleus, innervation may reach up to the cingulate cortex and the hippocampus with the dorsal raphe nucleus extending innervation to the substantia nigra, nucleus accumbens, amygdala, and striatum. The passage of drug toward and through any of these specified cerebral regions will influence the neuropharmacological response inherent to the respective serotonin receptor. To ascertain the serotonin-mediated functions outside and inside the brain, it is therefore important to quantify the levels of serotonin in these specified cerebral regions in addition to the total quantity of drug reaching the entire cerebral region (neuroavailability). This may help to estimate the "dose-neurotransmitterresponse" ratio. Therefore, in conclusion, the neurological function or applicability of a neuro-active drug should rather be determined by its "neurotransmittability" than solely oral bioavailability or neuroavailability.

\section{References}

1. Markou A, Chiamulera C, Geyer MA, Tricklebank M, Steckler T (2009) Removing obstacles in neuroscience drug discovery: the future path for animal models. Neuropsychopharmacology 34: 74-89.

*Corresponding author: Viness Pillay, University of the Witwatersrand, Faculty of Health Sciences, Department of Pharmacy and Pharmacology, 7 York Road Parktown, 2193, Johannesburg, South Africa, Tel: +27-11-717-2274; Fax: +27-11 642-4355; E-mail: viness.pillay@wits.ac.za

Received July 24, 2013; Accepted July 26, 2013; Published August 02, 2013

Citation: Pillay V, Kumar P, Choonara YE (2013) Oral Bioavailability and NeuroAvailability vs. "Neuro-Transmittability": A Figment of Imagination in the Conundrum of Neuronal Intervention. J Bioequiv Availab 5: e34. doi:10.4172/jbb.10000e34

Copyright: (c) 2013 Pillay V, et al. This is an open-access article distributed under the terms of the Creative Commons Attribution License, which permits unrestricted use, distribution, and reproduction in any medium, provided the original author and source are credited. 
Citation: Pillay V, Kumar P, Choonara YE (2013) Oral Bioavailability and Neuro-Availability vs. "Neuro-Transmittability": A Figment of Imagination in the Conundrum of Neuronal Intervention. J Bioequiv Availab 5: e34. doi:10.4172/jbb.10000e34

Page 2 of 2

2. Mas M, Gonzalez-Mora JL, Hernandez L (1996) In vivo monitoring of brain neurotransmitter release for the assessment of neuroendocrine interactions. Cell Mol Neurobiol 16: 383-396.

3. Ghersi-Egea JF, Monkkonen KS, Schmitt C, Honnorat J, Fèvre-Montange M, et al. (2009) Blood-brain interfaces and cerebral drug bioavailability. Rev Neurol (Paris) 165: 1029-1038
4. Murphy DL, Andrews AM, Wichems CH, Li Q, Tohda M, et al. (1998) Brain serotonin neurotransmission: an overview and update with an emphasis on serotonin subsystem heterogeneity, multiple receptors, interactions with other neurotransmitter systems, and consequent implications for understanding the actions of serotonergic drugs. J Clin Psychiatry 59: 4-12. 